\title{
A model of underlying socioeconomic vulnerability in human populations: evidence from variability in population health and implications for public health
}

\author{
Sandro Galea ${ }^{\mathrm{a}, \mathrm{b}, *}$, Jennifer Ahern ${ }^{\mathrm{a}}$, Adam Karpati ${ }^{\mathrm{c}}$ \\ ${ }^{a}$ Center for Urban Epidemiologic Studies, New York Academy of Medicine, 1216, 5th Avenue, Room 553, New York, NY 10029, USA \\ ${ }^{\mathrm{b}}$ Department of Epidemiology, Columbia University School of Public Health, USA \\ ${ }^{\mathrm{c}}$ New York City Department of Health and Mental Hygiene, USA
}

Available online 8 January 2005

\begin{abstract}
Drawing from insights into the variability of complex biologic systems we propose that the health of human populations reflects the interrelationship between underlying vulnerabilities (determined by population-level social and economic factors; e.g., income distribution) and capacities (determined by population-level salutary resources, e.g., social capital) and how populations, shaped by these vulnerabilities and capacities, respond to intermittent stressors (e.g., economic downturns) and protective events (e.g., introduction of a school). Monitoring this dynamic at the population-level can be accomplished by examining not only rates of illness and mortality, but variability in rates, either between populations or within populations over time. We used mortality data from New York City neighborhoods between 1990 and 2001 to test two related hypotheses consistent with this model of population health: (a) There is greater variability in mortality rates at a point in time between neighborhoods that are characterized by socioeconomic vulnerability; and (b) there is greater variability in mortality rates over time within neighborhoods that are characterized by socioeconomic vulnerability. We found that neighborhoods characterized by social and economic vulnerability displayed substantial variability in particular mortality rates. Mortality rates displaying the greatest variability were from causes that may be sensitive to social conditions (e.g., homicide or HIV/AIDS rates). Variability in population health existed both between neighborhoods with underlying vulnerability at one point in time and within vulnerable neighborhoods over time. The results of this analysis are consistent with a theory of underlying socioeconomic vulnerabilities of human populations and suggest that variability in population health may be an important consideration in population health assessment.
\end{abstract}

(C) 2004 Elsevier Ltd. All rights reserved.

Keywords: Variability; Vulnerability; Population health; Ecology; Neighborhood; USA

\footnotetext{
*Corresponding author. Center for Urban Epidemiologic Studies, New York Academy of Medicine, 1216, 5th Avenue, Room 553, New York, NY 10029, USA. Tel.: + 12128227378 ; fax: +12128766220 .

E-mail address: sgalea@nyam.org (S. Galea).
}

\section{Introduction}

In the field of ecology, the dynamics of groups of different species are studied as a means of allowing prediction of group behaviors and outcomes, both at equilibrium and in response to specific interventions (Levins, 1975). Although health-related empiric studies 
have traditionally focused on identifying individual-level characteristics that determine health, more recent work has shown that group characteristics may also importantly affect human health (O'Campo, 2003). For example, population-level socioeconomic status is associated with health-related behaviors independent of individual socioeconomic status (Subramanian, Kim, \& Kawachi, 2002). Also, it is increasingly recognized that simple linear cause and effect paradigms that assume that individuals are independent of one another are over-simplifications that fail to take into account patterns of connectedness among individuals (Koopman \& Lynch, 1999). For example, social network dynamics are associated with risk behavior and transmission of infectious diseases (Koopman \& Longini, 1994; Latkin, Forman, Knowlton, \& Sherman, 2003). Therefore, groups of individuals can be seen as population systems and the dynamics of human populations may determine health in their own right (Robert, 1999). Early theoretical and empiric work assessing the ecology of human health showed that characteristics of human populations, defined at the county level in two US states, are associated with population health in a manner similar to biological populations (Karpati, Galea, Awerbuch, \& Levins, 2002).

Complex systems are systems that are inadequately described by unidirectional causal relationships and that may require the consideration of multidirectional causal relationships (e.g., feedback) in order to permit both accurate description and prediction (Levins, 1974). Although it has long been accepted in ecology that population system dynamics are complex, there has been relatively less attention paid to the complexity of human population systems and how this complexity may shape population health. Several observations suggest that human populations behave as complex systems. First, there are multiple examples of discontinuous changes in health in relation to monotonic changes in exposures facing human populations (Philippe \& Mansi, 1998). For example, the relation between population health and several environmental exposures encompass threshold and sigmoid curves, both hallmarks of nonlinear dynamics (Maynard et al., 2003). Second, the effects of particular exposures on human populations can linger well beyond removal of the exposure. For example, the population mental health consequences of disasters are well known to persist beyond the disaster itself (Galea et al., 2003b,). Third, multiple diseases, including infectious diseases and neoplastic diseases, frequently share determinants that are affected by common environmental exposures (Koopman \& Lynch, 1999; Koopman \& Longini, 1994). Although none of these observations in and of themselves define complex systems, they provide empiric evidence that human populations exhibit complex system behaviors and that the application of ecologic principles may assist in describing, and understanding, population health.

Positing that human populations are complex systems whose properties are of empiric and potentially practical interest we hypothesize that the health of human populations reflects the interrelationship between underlying vulnerabilities and capacities and how populations, shaped by these vulnerabilities and capacities, respond to intermittent stressors and protective events. A wide range of factors may be considered to be underlying vulnerabilities, including a paucity of material resources (e.g., low income) available in a given human population, or the presence of a natural tectonic fault line that predisposes a population to earthquakes. Conversely, examples of underlying capacities may include social capital and abundant availability of natural resources. Intermittent stressors include the closure of a large employer, or a natural disaster. Conversely, protective events, such as the opening of a new school or an increase in group cohesiveness due to the success of a local sports team, also occur intermittently. Importantly, the intermittent influences interact with the underlying conditions to shape health at any particular moment. We note that intermittent stressors can be considered destabilizing phenomena while intermittent positive events may be stabilizing.

This model is a heuristic to explain how underlying and intermittent conditions may affect population health and of necessity represents a simplification. Therefore, while the model suggests that vulnerabilities and capacities are distinct constructs, the "absence of vulnerability" can be considered a capacity if we are comparing health indicators across different human populations. Similarly, the boundaries between intermittent "stressors" and "positive events" are simplifying devices intended to help explain the potential system dynamics that can affect population health. In addition, as noted in the examples given above, the range of factors that may shape population health may be "social and economic" factors, but also potentially "geographic", "climatologic" or a range of other categories that generally fall outside the realm of social epidemiology. Although we highlight here the role of underlying social and economic vulnerabilities we posit that this model of population health may be relevant to a range of potential vulnerabilities and capacities that extend beyond the focus of this paper.

Multiple academic disciplines have considered vulnerability as an important characteristic of both individuals and of populations (Bankoff, 2003; Turner et al., 2003; Cohen \& Hamrick, 2003). Although definitions of vulnerability vary in the scientific literature, it is generally considered to be the capacity for harm in an individual or system in response to a stimulus. It has been postulated that different elements of vulnerability exist, including genetic and biologic vulnerability at the 
individual level (Cohen \& Hamrick, 2003; Heath \& Nelson, 2002) and social vulnerability at the group level (McKeehan, 2000). Individuals who possess specific characteristics are frequently termed "vulnerable"; for example children, homeless persons, and minority innercity populations have been termed "vulnerable" in recent scientific publications suggesting they are more likely to be harmed by external stressors than are others in the general population (Stergiopoulos \& Herrmann, 2003; Shi, 2000). In the field of disaster preparedness, it has long been recognized that certain groups are more vulnerable to the effects of disasters than others. For example, wealthier communities are more likely to rebound from the consequences of natural disasters than less wealthy communities (Nelson, 1990). There is, in turn, complementary evidence that certain characteristics may confer protections on individuals or populations and may be considered capacities. For example, social capital has been shown to be associated with lower population mortality and potentially protects populations from the effects of income maldistribution (Kawachi, Kennedy, Lochner, \& Prothrow-Stith, 1997).

Having acknowledged human populations to be units of scientific interest and proposed a model that incorporates both underlying vulnerabilities/capacities and intermittent stressors/protective events, we can now consider how the interrelationship of these factors is reflected in the health of populations. Of particular interest to population health assessment is variability, a component of complex systems that reflects the impact of external stressors and the complex system's attempt at maintaining homeostasis (Levins \& Lopez, 1999). Variability of health indicators in human populations may be particularly informative in the study of underlying population vulnerability. In considering variability exhibited by ecologic systems, I.I. Schmalhausen observed that systems at the boundary of their tolerance are more vulnerable to small differences in circumstance and display more variability than systems not similarly stressed (Schmalhausen, 1949). Schmalhausen argued that through the process of biological evolution a species' phenotype is stable within the normal range of environmental variation. However, in extreme environmental conditions greater phenotypic variation manifests between organisms as characteristics of species that had not previously been a basis of selection are expressed. Extending Schmalhausen's observation, Levins and Lopez (1999) suggest that the impact of intermittent stressors will result in greater variability in outcome among vulnerable human populations than among populations that are not characterized by underlying vulnerability. Summarizing this argument, in populations with low levels of vulnerability (e.g., high income) the rates of disease and mortality would be expected to be stable as the population is resilient to changes that may occur in other conditions. However, in populations with high levels of vulnerability (e.g., low income) there would be greater variability in rates of disease and morality as characteristics of populations that are untested at low levels of vulnerability are expressed in the vulnerable state. For example, in wealthy populations, intermittent stressors may not affect health as the resources conferred by wealth keep disease and mortality rates constant. Conversely, in poor populations intermittent stressors or protective events may be critical in determining disease and mortality rates as there are fewer material protections available to the population. Variability is produced both by the random or uneven distribution of these intermittent events and by differences in underlying vulnerabilities and capacities. Therefore, returning to our model, intermittent stressors, or destabilizing events, affect a homeostatic system and produce varying degrees of change in the population system's properties; the variability in the change in system properties is a function of the extent to which the system is characterized by underlying vulnerabilities or capacities. For example, a geographically isolated community characterized by limited employment opportunities may not cope as well with the sudden departure of a major employer as a community where employment opportunities are abundant. However, the range of responses among populations with limited employment opportunities to the departure of a major employer may be broad and predicated on the distribution of population capacities, such as social capital, to help those newly unemployed. Conversely, the responses of populations characterized by abundant employment opportunities in the face of a departing employer are not dependent on the distribution of other capacities or vulnerabilities. As such, these populations' responses may be less variable than the responses of vulnerable populations. When examining human populations as population systems of interest, the variability exhibited by specific health indicators may provide evidence for underlying population vulnerability.

We used data from New York City (NYC) neighborhoods to test two hypotheses consistent with the model of population health proposed here: (a) There is greater variability in mortality rates at a point in time between neighborhoods that are characterized by socioeconomic vulnerability; and (b) there is greater variability in mortality rates over time within neighborhoods that are characterized by socioeconomic vulnerability. We note that the analysis shown here focuses on population vulnerability and that we do not formally assess the other elements (i.e., capacities, intermittent stressors, or protective events) of the proposed model. In addition we focus on social and economic factors as underlying vulnerabilities of interest (Marmot, Kogevinas, \& Elston, 1987; Geronimus, Bound, \& Waidmann, 1999; Adler et al., 1994; Sampson, Morenoff, \& Gannon- 
Rowley, 2002; Robert, 1999). We intend this analysis to illustrate how the behavior of human populations may be suggestive of complex system dynamics and to set the stage for further work that explicitly considers the relationships among underlying socioeconomic vulnerabilities and capacities and how these characteristics of human populations may assist in public health prediction.

\section{Methods \\ Units of analysis}

The units of analysis for this study were 59 neighborhoods in NYC. In considering the relevant population group that represents a system of interest it is desirable to identify units that are meaningful to their residents and that may plausibly shape residents' health and risk behavior. Existing research has utilized various definitions of neighborhoods, including communities as identified by their residents, block groups, census tracts, and clusters of census tracts (Curtis \& Rees Jones, 1998). Conceptually, there is no one neighborhood unit that is important to the exclusion of all other units. For example, while a person may be influenced by her immediate environment (few blocks) in choice of foods purchased, it is equally plausible that safety in the larger neighborhood determines whether the same person exercises on a regular basis. Previous research has shown that different social and environmental measures operate at different levels. For example, studies of social capital and health have been conducted at both the small neighborhood scale in Chicago and the statewide scale across the United States (see Sampson, Raudenbush, \& Earls, 1997; Kawachi, et al., 1997). Poverty at the state, county, city, and neighborhood levels has been linked to poor health status (Healy, 2003; Hillemeier, Lynch, Harper, Raghunathan, \& Kaplan, 2003; Marzuk et al., 1997). Appreciating that residents tend to interact with their neighbors and carry out activities within a physical environment that is close to their home, we used definitions of neighborhoods that are relevant to the conceptual model guiding our research. In this analysis, we use the 59 NYC community districts as proxies for neighborhoods. Community districts are well-defined units, each with an administrative community board, that as such have political and social a priori significance for their residents. Political and social decisions by community boards may have an impact on the factors that are considered here to characterize underlying neighborhood vulnerability. For example, community boards may make decisions that influence school quality and placement and hence educational attainment in a given neighborhood. Community districts roughly correspond to aggregations of census tracts, and were initially defined by a resident consultative process organized by the Office of City Planning to reflect residents' own descriptions of neighborhoods in the 1970s. Although the community districts are not demographically homogenous (as would be expected in a city as diverse as NYC), they represent neighborhoods that have been shown to affect resident behavior and health (Tardiff, Gross, \& Messner, 1986; Marzuk et al., 1997; Galea et al., 2003a). Based on the 2000 Census, the 59 NYC community districts have a mean of 135,681 residents $($ median $=128,313$; interquartile range $=104,358-167,005 ;$ range $=34,420-242,952$ ).

\section{Data}

We used data from the New York City Department of Health and Mental Hygiene, Bureau of Vital Statistics to calculate age-adjusted mortality rates in each New York City neighborhood for 12 different causes, between 1990 and 2001. For data between 1990 and 1998, ICD-9 coded underlying cause of death were used; ICD-10 codes were used between 1999 and 2001. Causes of mortality studied were: cardiovascular disease (ICD-9 393-398, 402, 404-429; ICD-10 I00-I09, I11, I13, I20I51), malignant neoplasms (ICD-9 140-208; ICD10 C00C97), accidents (ICD-9 E800-E849; ICD10 V01-X59, Y85-Y86), chronic lower respiratory disease (ICD-9 490-496; ICD-10 J40-J47), cerebrovascular disease (ICD-9 430-438; ICD-10 I60-I69), pneumonia and influenza (ICD-9 480-487; ICD-10 J10-J18), diseases of the nervous system (ICD-9 320-389; ICD-10 G00-G98), suicide (ICD-9 E950-E959; ICD-10 X60-X84, Y87.0), chronic liver disease and cirrhosis (ICD-9 571; ICD-10 K70, K73-74), diabetes (ICD-9 250; ICD-10 E10-E14), homicide (ICD-9 E960-E975, E990-E999; ICD-10 X85Y09, Y87.1) and HIV/AIDS (ICD-9 043-044; ICD-10 B20-B24). For year-, neighborhood-, and age-specific denominators, we used US Census data from 1990 and 2000 and calculated inter-censal counts using linear interpolation. We aggregated census tract data from the US Bureau of the Census to obtain information about three potential markers of neighborhood vulnerability: neighborhood income, income distribution, and education. We used median household income to assess neighborhood income, the Gini coefficient to assess income distribution (Subramanian \& Kawachi, 2004), and the percent of adults with a less than high school education to assess neighborhood education.

\section{Analysis}

First, we considered variability in mortality rates between neighborhoods at one point in time by analyzing 2000 data. Second, we considered variability in yearly mortality rates within neighborhoods from 1990 to 2001. 


\section{Inter-neighborhood variability 2000}

We calculated the variability of each health indicator across all community districts in New York City in 2000. We used three measures of variability in our analyses, all quantifying the absolute variability of the disease rate while accounting for the mean to ensure that apparent cross-sectional differences in variability of mortality rates from different causes were not due to differences in average mortality rates between causes. Measures of variability in this cross-sectional analysis were: the interquartile range (IQR) divided by the mean, the range of values divided by the mean, and the coefficient of variation (i.e., the standard error divided by the mean). Examining the variance divided by the mean would not be appropriate in this context as the variance has units that are squared, while the mean has units on the original scale of the mortality rate.

To quantify differences in inter-neighborhood variability in 2000 by different markers of vulnerability, we utilized the Breusch-Pagan test for heteroskedasticity (Breusch \& Pagan, 1979). The test is performed by first conducting a linear regression in which the measure of vulnerability predicts the mortality rate outcome. The residuals from this first model are squared. In a second linear regression model the measure of vulnerability is used to predict the squared residuals of the first model which quantify the variability in the outcome. A positive association indicates that at increasing levels of vulnerability there is more variability in the disease outcome. An $F$-test is then used to determine whether there is a significant association between the measure of vulnerability and the variability in the outcome (as quantified by the squared residuals):

$F_{(\mathrm{df}=k, n-k-1)}=\left[\frac{R^{2}}{k}\right] /\left[\frac{\left(1-R^{2}\right)}{(n-k-1)}\right]$,

where $R^{2}$ is from the second model, $n$ is the number of observations, and $k$ is the number of predictors in the model ( 1 in our analyses). A significant finding $(p<0.05)$ indicates heteroskedasticity.

To visually illustrate inter-neighborhood variability in mortality rates in 2000 we created plots of neighborhood mortality rates by neighborhood vulnerability measures. Examples of mortality rates with high and low variability are presented, using neighborhood education as the measure of vulnerability. In these plots, a larger spread in disease rates at higher levels of vulnerability illustrates the variability of interest.

\section{Intra-neighborhood variability over time, 1990-2001}

We summarized the intra-neighborhood variability of the age-adjusted mortality rates for each neighborhood between 1990 and 2001 using the IQR divided by the mean, the range of values divided by the mean, and the coefficient of variation (i.e., the standard error divided by the mean). For each neighborhood and cause of mortality, the distribution of these measures of variability was presented in box plots. The IQR divided by the mean is used for illustration.

To examine whether variable outcomes have more pronounced variability in vulnerable neighborhoods we assessed associations between intra-neighborhood variability and measures of vulnerability using Pearson correlation coefficients between the three socioeconomic vulnerabilities in 1990 and measures of variability over time for each mortality rate of interest. We used the IQR, range, and standard deviation to assess variability in each cause of mortality for each neighborhood over time. In this analysis, division of variability measures by the mean is no longer appropriate since we are interested in a summary of yearly variability in one cause of mortality within neighborhoods, rather than comparing variability between causes of mortality.

To visually illustrate intra-neighborhood variability in mortality rates from 1990 to 2001 by different levels of vulnerability, we created plots of variability in mortality rates over time by neighborhood vulnerability measures. Examples of outcomes with high and low variability are presented, using neighborhood education as the measure of vulnerability. In these plots, a steeper slope in the association between measures of disease rate variability and measures of vulnerability illustrates the variability of interest.

\section{Results}

Inter-neighborhood variability, 2000

Measures of variability in the inter-neighborhood ageadjusted mortality rates in 2000 are presented in Table 1; the table is sorted by increasing variability based on the $\mathrm{IQR} /$ mean.

Certain causes of death had more variability between neighborhoods in 2000; the highest variability in outcome was exhibited by HIV/AIDS mortality (interquartile range $(\mathrm{IQR}) /$ mean $=1.34$; range $/$ mean $=4.24$; coefficient of variation $=0.96$ ), and homicide mortality $(\mathrm{IQR} /$ mean $=1.27$; range $/$ mean $=2.98$; coefficient of variation $=1.77)$. The lowest variability was exhibited by cardiovascular disease mortality $(\mathrm{IQR} / \mathrm{mean}=0.21$; range $/$ mean $=1.34$; coefficient of variation $=0.19)$ and malignant neoplasm mortality $(\mathrm{IQR} /$ mean $=0.27$; range $/$ mean $=0.84$; coefficient of variation $=0.18$ ).

To examine inter-neighborhood variability in 2000 at different levels of vulnerability we tested for heteroskedasticity in the mortality rates by neighborhood income, Gini, and education. As shown in Table 1, by income there was significant heteroskedasticity in the 
Table 1

Variability in mortality rates between neighborhoods and heteroskedasticity in mortality rates by measures of vulnerability; New York City, 2000

\begin{tabular}{|c|c|c|c|c|c|c|c|c|c|c|c|c|c|}
\hline \multirow[t]{2}{*}{ Mortality rate } & \multirow[t]{2}{*}{ Mean } & \multirow[t]{2}{*}{ IQR } & \multirow[t]{2}{*}{ Range } & \multirow[t]{2}{*}{$\begin{array}{l}\text { Standard } \\
\text { deviation }\end{array}$} & \multirow[t]{2}{*}{$\begin{array}{l}\mathrm{IQR} / \\
\text { mean }\end{array}$} & \multirow[t]{2}{*}{$\begin{array}{l}\text { Range/ } \\
\text { mean }\end{array}$} & \multirow[t]{2}{*}{$\begin{array}{l}\text { Coefficient } \\
\text { of variation }^{\mathrm{a}}\end{array}$} & \multicolumn{2}{|c|}{$\begin{array}{l}\text { Heteroskedasticity by } \\
\text { income }^{b}\end{array}$} & \multicolumn{2}{|c|}{$\begin{array}{l}\text { Heteroskedasticity } \\
\text { by Gini }{ }^{\mathrm{c}}\end{array}$} & \multicolumn{2}{|c|}{$\begin{array}{l}\text { Heteroskedasticity } \\
\text { by education }{ }^{\mathrm{d}}\end{array}$} \\
\hline & & & & & & & & $F^{\mathrm{e}}$ & $p$-value & $F$ & $p$-value & $F$ & $p$-value \\
\hline $\begin{array}{l}\text { Cardiovascular } \\
\text { disease }\end{array}$ & 310.48 & 64.41 & 415.14 & 59.82 & 0.21 & 1.34 & 0.19 & 0.06 & 0.8019 & 0.25 & 0.6156 & 0.44 & 0.508 \\
\hline $\begin{array}{l}\text { Malignant } \\
\text { neoplasm }\end{array}$ & 173.23 & 46.51 & 145.90 & 30.57 & 0.27 & 0.84 & 0.18 & 2.38 & 0.1287 & 1.84 & 0.1804 & 1.58 & 0.2133 \\
\hline Accidents & 12.41 & 4.61 & 14.25 & 3.33 & 0.37 & 1.15 & 0.27 & 0.7 & 0.4047 & 0.01 & 0.9226 & 0.36 & 0.5524 \\
\hline $\begin{array}{l}\text { Chronic lower } \\
\text { respiratory disease }\end{array}$ & 21.96 & 9.71 & 32.42 & 8.37 & 0.44 & 1.48 & 0.38 & 0.01 & 0.9158 & 0.13 & 0.7155 & 0.71 & 0.4024 \\
\hline $\begin{array}{l}\text { Cerebrovascular } \\
\text { disease }\end{array}$ & 26.59 & 15.41 & 36.24 & 9.50 & 0.58 & 1.36 & 0.36 & 0.48 & 0.4923 & 13.51 & 0.0005 & 0.27 & 0.6046 \\
\hline $\begin{array}{l}\text { Pneumonia and } \\
\text { influenza }\end{array}$ & 30.10 & 17.78 & 48.70 & 12.11 & 0.59 & 1.62 & 0.40 & 0.55 & 0.4629 & 0.06 & 0.8038 & 0.39 & 0.5331 \\
\hline $\begin{array}{l}\text { Disease of the } \\
\text { nervous system }\end{array}$ & 8.70 & 5.53 & 19.84 & 4.15 & 0.64 & 2.28 & 0.48 & 1.09 & 0.3015 & 6.33 & 0.0147 & 0.99 & 0.3237 \\
\hline Suicide & 5.15 & 3.36 & 11.34 & 2.35 & 0.65 & 2.20 & 0.46 & 0.89 & 0.3498 & 0.15 & 0.698 & 2.31 & 0.1337 \\
\hline $\begin{array}{l}\text { Chronic liver } \\
\text { disease and cirrhosis }\end{array}$ & 8.02 & 6.35 & 33.67 & 6.10 & 0.79 & 4.20 & 0.76 & 3.76 & 0.0575 & 3.86 & 0.0545 & 3.94 & 0.052 \\
\hline Diabetes & 28.45 & 26.18 & 98.70 & 19.54 & 0.92 & 3.47 & 0.69 & 3.09 & 0.0843 & 2.73 & 0.1041 & 4.1 & 0.0475 \\
\hline Homicide & 8.39 & 10.63 & 24.98 & 6.42 & 1.27 & 2.98 & 0.77 & 8.78 & 0.0044 & 8.81 & 0.0044 & 14.26 & 0.0004 \\
\hline HIV/AIDS & 29.41 & 39.51 & 124.57 & 28.16 & 1.34 & 4.24 & 0.96 & 2.27 & 0.1378 & 5.47 & 0.0229 & 2.95 & 0.0916 \\
\hline
\end{tabular}

${ }^{\mathrm{a}}$ Coefficient of variation: standard deviation/mean.

${ }^{\mathrm{b}}$ Median income in 2000 from the US Census.

${ }^{\mathrm{c}}$ Gini coefficient measuring income inequality in 2000 from the US Census.

${ }^{\mathrm{d}}$ Percent of adults with a less than high school education in 2000 from the US Census.

${ }^{\mathrm{e}}$ Breusch-Pagan $F$-tests (Breusch and Pagan, 1979). 
rate of homicide mortality $(p=0.004)$, and trends suggesting heteroskedasticity for the rates of diabetes $(p=0.08)$ and liver disease $(p=0.06)$ mortality. By the Gini coefficient, there was significant heteroskedasticity in the rates of HIV/AIDS $(p=0.02)$, homicide $(p=0.004)$, nervous system disease $(p=0.01)$, and cerebrovascular disease $(p=0.0005)$ mortality, and there was a trend suggesting heteroskedasticity for the rate of liver disease mortality $(p=0.055)$. By education, there was significant heteroskedasticity in the rates of homicide $(p=0.0004)$ and diabetes $(p=0.048)$ mortality, and there were trends suggesting heteroskedasticity in the rates of HIV/AIDS $(p=0.09)$ and liver disease $(p=0.052)$ mortality.

To provide illustrations of the associations between measures of vulnerability and levels of variability in mortality rates, we present scatterplots of selected population-level mortality rates (homicide and stroke) and a measure of vulnerability (the proportion of adult neighborhood residents with less than a high school education). Homicide mortality, as shown above, has high variability across neighborhoods and cerebrovascular disease mortality is in the middle of the range of variability levels (Table 1). In the scatterplot showing the association between neighborhood age-adjusted homicide rates and neighborhood education (Fig. 1) we observe that at the low levels of vulnerability (i.e., where the proportion of adults with less than a high school education is low), the variability in homicide rates is also low. Conversely, among neighborhoods with high levels of vulnerability (i.e., where the proportion of adults with less than a high school education is high), the variability in homicide rates was also high. The test of heteroskedasticity in Table 1 demonstrates that the greater variability in homicide rates observed at higher levels of low education is statistically significant $(F=14.26, p=0.0004)$.

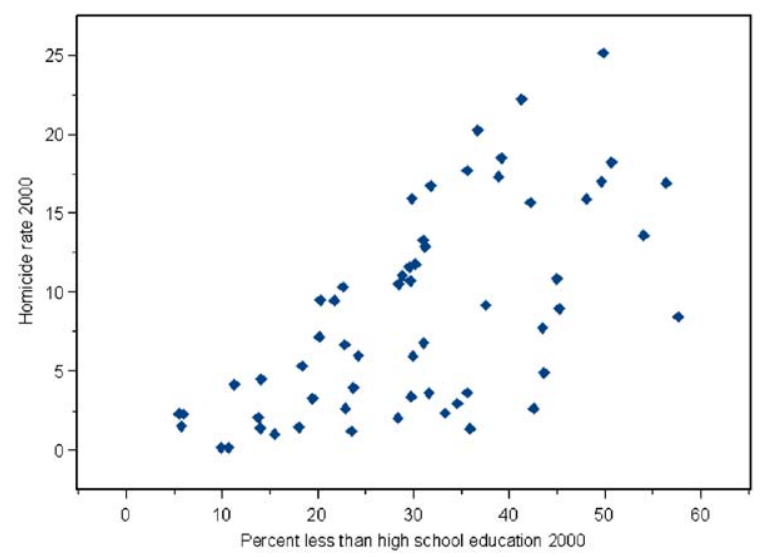

Fig. 1. Scatterplot of age-adjusted homicide mortality rates and the proportion of adult residents in the neighborhood with less than a high school education; New York City, 2000.

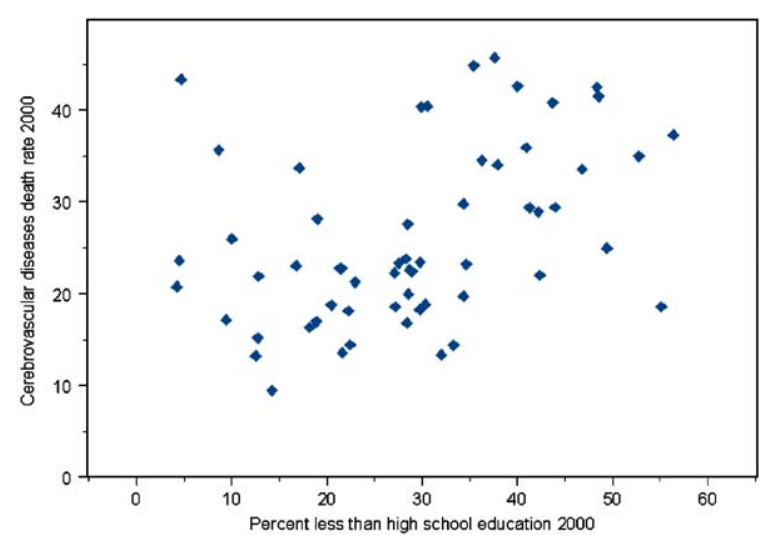

Fig. 2. Scatterplot of the age-adjusted cerebrovascular disease mortality rates and proportion of adult residents in the neighborhood with less than a high school education; New York City, 2000.

In contrast, the scatterplot of neighborhood ageadjusted cerebrovascular disease rates and education (Fig. 2) shows no comparable increase in variability in cerebrovascular disease mortality at high levels of ecologic vulnerability; the variability among neighborhood cerebrovascular disease mortality rates was comparable for neighborhoods with low and high proportions of adults with less than a high school education. Based on the test of heteroskedasticity, there is no significant difference in the variability of cerebrovascular disease mortality rates by levels of neighborhood education $(F=0.27, p=0.6)$.

\section{Intra-neighborhood variability over time, 1990-2001}

We first assessed correlations between each of the three measures of vulnerability from 1990 and 2000; the correlation between proportion of adults with less than a high school education in 1990 and 2000 was 0.98 , the correlation between median household income in 1990 and 2000 was 0.96 , and the correlation between Gini coefficients in 1990 and 2000 was 0.94 , suggesting that the underlying vulnerabilities being studied change slowly over time. When evaluating intra-neighborhood temporal variability we used vulnerability measures assessed in 1990 .

To assess differences in temporal variability in mortality rates by cause, we compared intra-neighborhood variability in age-adjusted mortality rates 1990-2001 across causes of mortality (Fig. 3). The variability in mortality rates over the decade was greater on average for causes of mortality such as HIV/AIDS and homicide and lower for causes of mortality such as cardiovascular disease and malignant neoplasms.

The correlations between intra-neighborhood variability in each yearly neighborhood mortality rate 


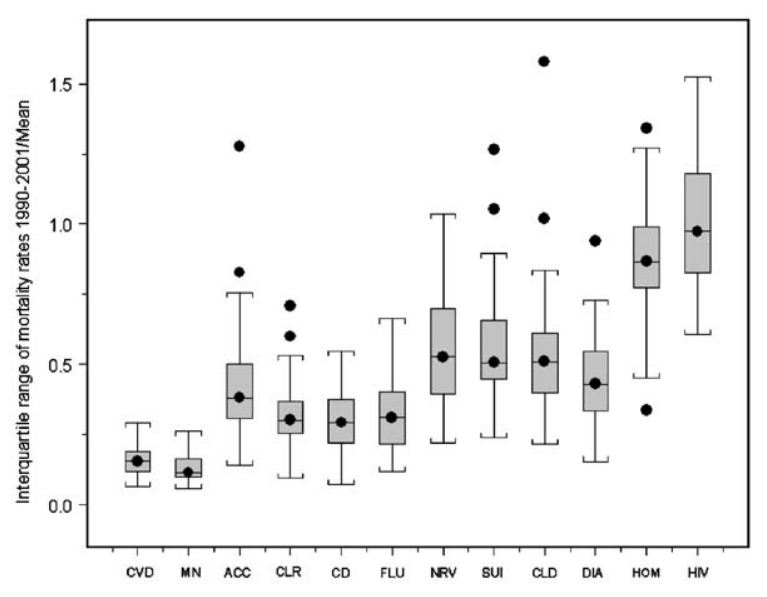

Fig. 3. Box plots of the interquartile range/mean of ageadjusted mortality rates from 12 causes 1990-2001 across New York City neighborhoods. CVD: cardiovascular disease, MN: malignant neoplasm, ACC: accidents, CLR: chronic lower respiratory disease, CD: cerebrovascular, disease, FLU: pneumonia and influenza, NRV: disease of the nervous system, SUI: suicide, CLD: chronic liver disease and cirrhosis, DIA: diabetes, HOM: homicide, HIV: HIV/AIDS.

between 1990 and 2001 and each of the measures of vulnerability are presented in Table 2. Generally, stronger correlations were found between measures of vulnerability and variability in specific health indicators. For example, the absolute values of the correlations between measures of vulnerability and level of variability ranged from 0.51 to 0.81 for homicide, from 0.42 to 0.69 for diabetes, from 0.55 to 0.74 for liver disease. In contrast, the absolute values of the correlations between measures of vulnerability and level of variability ranged from 0.22 to 0.59 for cardiovascular disease, and from 0.18 to 0.34 for malignant neoplasms.

Scatterplots visually illustrating the correlations between measures of vulnerability and level of variability are presented in Figs. 4 and 5. Fig. 4 presents the association between the IQR of intra-neighborhood yearly homicide rates between 1990 and 2001 and the proportion of adults in the neighborhood with less than a high school education in 1990. Assessment of variability in yearly homicide rates within neighborhoods shows that at the low levels of vulnerability (i.e., where the proportion of adults with less than a high school education is low), the intra-neighborhood variability in yearly homicide rates was also low. Conversely, among neighborhoods with high levels of vulnerability (i.e., where the proportion of adults with less than a high school education is high), the intra-neighborhood variability in yearly homicide rates was also high. It is important to emphasize that each value on the $y$-axis quantifies variability in rates (in contrast to Figs. 2 and 3 where the $y$-axis quantifies rates). Thus, the associations between measures of vulnerability and variability in the mortality rates are illustrated in the magnitude of the upward trend in the scatterplot, rather than the variability around the general trend. Inspection of the scatterplot shown in Fig. 4 reveals an upward trend with lower intra-neighborhood variability in yearly homicide rates giving way to higher intra-neighborhood variability in yearly homicide rates with increasing levels of vulnerability. The correlation for this association of 0.80 (Table 2) suggests a strong relation.

Fig. 5 presents a scatterplot of the association between intra-neighborhood variability in yearly cerebrovascular disease death rate and neighborhood education. The intra-neighborhood variability in yearly cerebrovascular disease mortality rates was similar at all levels of neighborhood education. The correlation for this association of 0.37 (Table 2) suggests that the relation is not particularly strong.

\section{Discussion}

This analysis of mortality rates in NYC neighborhoods showed that variability in mortality rates, both between neighborhoods at one point in time and within neighborhoods over time, was greater in neighborhoods characterized by socioeconomic vulnerability. These findings are consistent with ecologic principles and suggest parallel processes underlying the socioeconomic vulnerabilities of human populations.

The notion of underlying socioeconomic vulnerability in human populations in some ways reflects theories of vulnerability in individuals and in individual physiologic systems that previously have been posited. Money, power, and prestige have been described as "fundamental causes" of individual human health; this can be considered an individual-level analogue to the arguments being proposed here (Link \& Phelan, 2000). Link and Phelan (2000) have argued that these socioeconomic factors are inalienable determinants of human health and contribute to the persistent disparities in individual health along racial and economic lines. Similarly, with respect to vulnerability of physiologic systems, it has been suggested that parameters such as chronic stress affect adrenal capacity and make the hypothalamopituitary-adrenal axis, and effectively the individual, more vulnerable to intermittent stressors. Our conception of vulnerability at the population level, however, suggests that there are vulnerabilities that predispose human populations to variability in population health. The implication of this observation is that population health cannot be determined by considering only the dynamics that affect individual health. In postulating that human populations are units of practical interest we suggest that the study of population health is incomplete 
Table 2

Correlations between variability in mortality rates within neighborhoods over time and measures of vulnerability; New York City, 1990-2001

\begin{tabular}{|c|c|c|c|c|c|c|c|c|c|}
\hline \multirow[t]{2}{*}{$\begin{array}{l}\text { Mortality rate variability } \\
1990-2000\end{array}$} & \multicolumn{3}{|c|}{$\begin{array}{l}\text { Mortality rate variability } 1990-2000 \\
\text { measured with IQR }\end{array}$} & \multicolumn{3}{|c|}{$\begin{array}{l}\text { Mortality rate variability 1990-2000 measured } \\
\text { with range }\end{array}$} & \multicolumn{3}{|c|}{$\begin{array}{l}\text { Mortality rate variability } 1990-2000 \text { measured } \\
\text { with standard deviation }\end{array}$} \\
\hline & Income $^{\mathrm{a}}$ & Gini $^{b}$ & Education $^{\mathrm{c}}$ & Income & Gini & Education & Income & Gini & Education \\
\hline \multirow[t]{2}{*}{ Cardiovascular disease } & $-0.43^{\mathrm{d}}$ & 0.22 & 0.51 & -0.57 & 0.38 & 0.59 & -0.56 & 0.33 & 0.59 \\
\hline & $0.0007^{\mathrm{e}}$ & 0.0879 & $<0.0001$ & $<0.0001$ & 0.003 & $<0.0001$ & $<0.0001$ & 0.0096 & $<0.0001$ \\
\hline \multirow[t]{2}{*}{ Malignant neoplasm } & -0.24 & 0.18 & 0.26 & -0.34 & 0.24 & 0.36 & -0.33 & 0.23 & 0.34 \\
\hline & 0.0674 & 0.1812 & 0.0475 & 0.0077 & 0.0723 & 0.0046 & 0.0104 & 0.0772 & 0.008 \\
\hline \multirow[t]{2}{*}{ Accidents } & -0.64 & 0.47 & 0.60 & -0.64 & 0.43 & 0.63 & -0.68 & 0.47 & 0.67 \\
\hline & $<0.0001$ & 0.0001 & $<0.0001$ & $<0.0001$ & 0.0006 & $<0.0001$ & $<0.0001$ & 0.0002 & $<0.0001$ \\
\hline \multirow{2}{*}{$\begin{array}{l}\text { Chronic lower respiratory } \\
\text { disease }\end{array}$} & -0.37 & 0.27 & 0.43 & -0.37 & 0.33 & 0.39 & -0.37 & 0.33 & 0.40 \\
\hline & 0.0035 & 0.041 & 0.0008 & 0.0041 & 0.0099 & 0.0025 & 0.004 & 0.0098 & 0.0019 \\
\hline \multirow[t]{2}{*}{ Cerebrovascular disease } & -0.42 & 0.40 & 0.37 & -0.47 & 0.51 & 0.45 & -0.49 & 0.51 & 0.46 \\
\hline & 0.0009 & 0.0019 & 0.0037 & 0.0002 & $<0.0001$ & 0.0004 & $<0.0001$ & $<0.0001$ & 0.0003 \\
\hline \multirow[t]{2}{*}{ Pneumonia and influenza } & -0.55 & 0.51 & 0.50 & -0.58 & 0.49 & 0.54 & -0.59 & 0.52 & 0.55 \\
\hline & $<0.0001$ & $<0.0001$ & $<0.0001$ & $<0.0001$ & $<0.0001$ & $<0.0001$ & $<0.0001$ & $<0.0001$ & $<0.0001$ \\
\hline \multirow{2}{*}{$\begin{array}{l}\text { Disease of the nervous } \\
\text { system }\end{array}$} & -0.20 & 0.39 & 0.18 & -0.19 & 0.44 & 0.18 & -0.15 & 0.42 & 0.15 \\
\hline & 0.1382 & 0.0025 & 0.1821 & 0.1606 & 0.0005 & 0.1696 & 0.2566 & 0.001 & 0.2437 \\
\hline \multirow[t]{2}{*}{ Suicide } & -0.12 & 0.35 & 0.08 & -0.28 & 0.38 & 0.26 & -0.25 & 0.41 & 0.22 \\
\hline & 0.3488 & 0.0061 & 0.5357 & 0.0336 & 0.003 & 0.0478 & 0.0583 & 0.0013 & 0.1001 \\
\hline \multirow{2}{*}{$\begin{array}{l}\text { Chronic liver disease and } \\
\text { cirrhosis }\end{array}$} & -0.71 & 0.55 & 0.68 & -0.74 & 0.57 & 0.73 & -0.76 & 0.59 & 0.74 \\
\hline & $<0.0001$ & $<0.0001$ & $<0.0001$ & $<0.0001$ & $<0.0001$ & $<0.0001$ & $<0.0001$ & $<0.0001$ & $<0.0001$ \\
\hline \multirow[t]{2}{*}{ Diabetes } & -0.62 & 0.42 & 0.67 & -0.65 & 0.46 & 0.68 & -0.65 & 0.46 & 0.69 \\
\hline & $<0.0001$ & 0.0011 & $<0.0001$ & $<0.0001$ & 0.0002 & $<0.0001$ & $<0.0001$ & 0.0003 & $<0.0001$ \\
\hline \multirow[t]{2}{*}{ Homicide } & -0.81 & 0.54 & 0.80 & -0.79 & 0.51 & 0.79 & -0.80 & 0.52 & 0.80 \\
\hline & $<0.0001$ & $<0.0001$ & $<0.0001$ & $<0.0001$ & $<0.0001$ & $<0.0001$ & $<0.0001$ & $<0.0001$ & $<0.0001$ \\
\hline \multirow[t]{2}{*}{ HIV/AIDS } & -0.43 & 0.69 & 0.29 & -0.55 & 0.71 & 0.43 & -0.50 & 0.71 & 0.37 \\
\hline & 0.0006 & $<0.0001$ & 0.0233 & $<0.0001$ & $<0.0001$ & 0.0007 & $<0.0001$ & $<0.0001$ & 0.0043 \\
\hline
\end{tabular}

${ }^{\mathrm{a}}$ Median income in 1990 from the US Census.

${ }^{\mathrm{b}}$ Gini coefficient measuring income inequality in 1990 from the US Census.

${ }^{c}$ Education: percent of adults with a less than high school education in 1990 from the US Census.

${ }^{\mathrm{d}}$ Pearson correlation coefficient.

${ }^{\mathrm{e}} p$-value. 


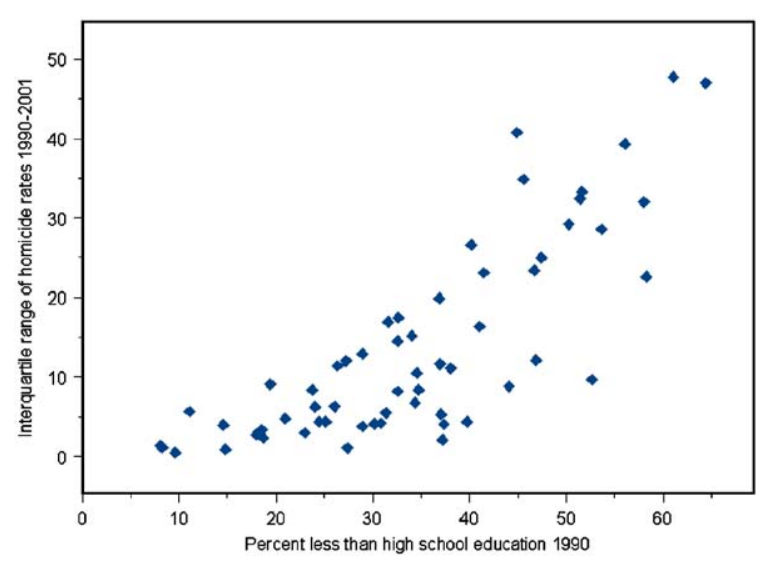

Fig. 4. Scatterplot of the interquartile range of age-adjusted homicide mortality rates in each New York City neighborhood 1990-2001 and the proportion of adult residents in the neighborhood with less than a high school education in 1990 .

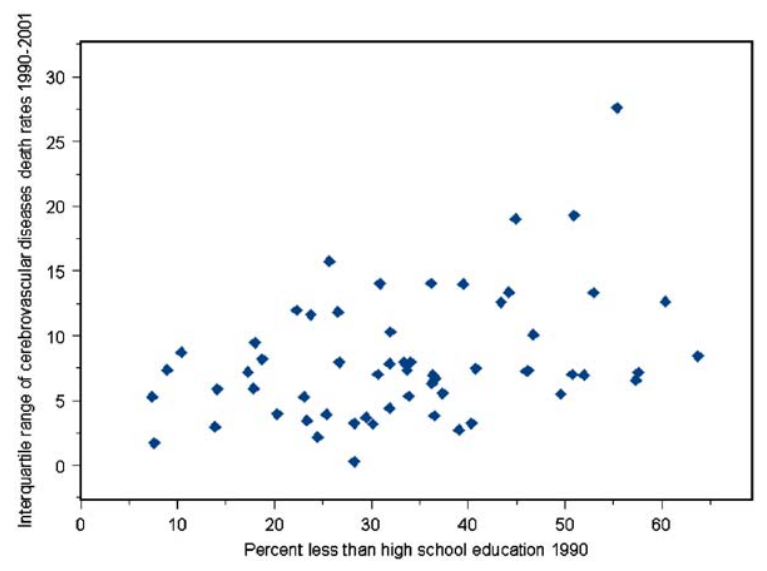

Fig. 5. Scatterplot of the interquartile range of age-adjusted cerebrovascular disease mortality rates in each New York City neighborhood 1990-2001 and the proportion of adult residents in the neighborhood with less than a high school education in 1990.

unless determinants of population health apart from the individual are considered. Athough we used aggregate education and income levels in this analysis we consider these variables to be proxies for characteristics of neighborhoods that go beyond the aggregation of individual characteristics. Aggregate education and income reflect an underlying neighborhood socioeconomic status that has implications for neighborhood conditions, resources, and social services that extend beyond any individual's contribution to these conditions. Income distribution is perhaps an easier example to consider since distribution of income is not an aggregate characteristic of individuals but rather a property of populations themselves. The proposed underlying socioeconomic vulnerability of human populations is conceived as a characteristic of the populations themselves and as such is influenced by stressors and positive events at the population level. These influences on human populations result in differences in the amount of cross-sectional and temporal variability in population health.

The model of underlying vulnerabilities and capacities proposed here, and the methods and examples used for illustration, are intended to illuminate a conceptual framework that may be useful in explaining population health. However, this work is only an early illustration and there are several considerations, and avenues of research, that would need to be explored to develop this heuristic further and to extend the observations documented in this paper.

We observed that there was more variability for certain mortality rates. For example, the variability in specific health indicators like homicide or HIV/AIDS mortality rates was consistently higher than the variability in specific health indicators like malignant neoplasm or chronic lower respiratory disease mortality rates. One explanation for this observation is that specific health indicators are more sensitive to social conditions than others. For example, exercise patterns, nutrition, and disease self-management, all of which may be influenced by material and social deprivation, contribute to population diabetes mortality rates. In contrast, endogenous influences (e.g., genetic factors) may more strongly determine mortality rates from malignant neoplasm.

We have focused in this analysis only on underlying population vulnerabilities although we postulate that the observed variability reflects the presence of underlying capacities and intermittent stressors/protective events as equally important determinants of population dynamics and population health. We chose here to focus on vulnerabilities in order to build on a body of literature in public health that considers the importance of vulnerability as a construct and to demonstrate a relation between socioeconomic vulnerability and population health in particular. However, further work would need to explore the presence of capacities hypothesized in our heuristic model. Also, we consider here three population-level socioeconomic factors interchangeably, i.e., two compositional measures (aggregates of individual socioeconomic status measures, i.e., income and education) and one contextual measure (a measure that has no individual-level analog, i.e., income distribution). While by and large our analyses show that the three do behave similarly, there are differences in heteroskedasticity of population health indicators modeled to reflect different vulnerabilities that suggest that the three constructs might act differently for different health indicators. For 
example, while certain health indicators (e.g., homicide) are highly heteroskedastic when modeled in relation to all three vulnerabilities considered, specific indicators, such as nervous system diseases are more heteroskedastic when modeled in relation to the Gini coefficient. It would not be surprising that different socioeconomic vulnerabilities may be relevant in different contexts. For example, while overall low neighborhood socioeconomic status may reflect a paucity of material resources available to specific populations, high income inequality may reflect both psychosocial stressors and disinvestment in material and human resources (Kaplan, Pamuk, Lynch, Cohen, \& Balfour, 1996); these differences suggest that the vulnerabilities discussed here may prove to play different roles in the complex dynamics that ultimately determine population health. Further work should assess the role of different socioeconomic vulnerabilities in population dynamics and potentially consider contextual characteristics that are determinants of population health that do not represent aggregates of individual measures. As a corollary to this observation we note that although, by and large, health indicators that were heteroskedastic in relation to markers of socioeconomic vulnerability were also the most variable health indicators within neighborhoods over time, our data reveals exceptions. For example, there is more variability in cerebrovascular disease over time than there is cross-sectionally. Although these data are too limited to draw inference from these observations it is plausible that the role of different socioeconomic vulnerabilities may be different between neighborhoods or within neighborhoods over time or that different diseases may manifest variability on different time scales.

Human populations may be characterized by multiple groupings and there are several other population groups that may be relevant and whose dynamics may affect overall population health. Although a growing body of empiric work, including this paper, consider geographically defined populations as units of interest (in this case we used neighborhoods as the geographic unit of interest although an extensive social epidemiologic literature has employed other geographic units including census tracts, zip codes, counties, and states among others), the observations drawn here may extend to other human populations. For example, in certain contexts (e.g., within a particular city) the connections among individuals within racially defined groups (e.g., persons of Caribbean descent) also may influence the health of members of these groups and as such, the observations described here may apply equally well to these racially defined population groups. Individuals may then belong to several populations, each of which may be important units of analysis. It is clear that any single consideration of individual group membership is of necessity a simplification of a rather more complicated picture that involves multiple, potentially overlapping, population groups. It is the purpose of this paper to provide an early illustration of the application of ecologic methods to the study of population health by considering geographically defined human populations; further work would need to consider the implications of multiple and overlapping populations and their role in determining population health.

Although we suggest that vulnerabilities and capacities are distinct entities, as discussed earlier, underlying characteristics of populations can equally be conceived as lying on a spectrum of vulnerability-capacity and behaving differently in response to different external events. Also, it is likely that there exists extensive feedback between the vulnerabilities, capacities, stressors, and positive events discussed here and that they modify one another. For example, a population faced with repeated economic stressors (e.g., economic downturns, loss of job-generating industry) will eventually undergo a change in its underlying socioeconomic vulnerability making it in turn more susceptible to the impact of intermittent stressors.

Work needs to be done to identify the relevant vulnerabilities and capacities for different human populations at different levels of aggregation. Human populations characterized at the country, neighborhood, or the social network level probably have different characteristics, and different socioeconomic vulnerabilities may be relevant determinants of group health. Although in this paper we present three forms of socioeconomic vulnerability for the purposes of illustration and show that population health, in the presence of each of these vulnerabilities, exhibits comparable variability, this does not mean that the three vulnerabilities are interchangeable or independent. Neighborhoods may be characterized by maldistributed income and low socioeconomic status or by maldistributed income and high socioeconomic status. It is likely that the combination of different vulnerabilities is a relevant consideration and potentially imposes a level of vulnerability on a neighborhood that extends beyond the effect of specific socioeconomic factors in isolation.

We employ here simple analytic methods to illustrate heterogeneity in population variability in mortality rates between neighborhoods at one point in time and within neighborhoods over time. In so doing we focus on only one element of what we suggest is a much more complex dynamic shaping population health. We document heterogeneity in mortality rates between neighborhoods in our cross-sectional analysis and within neighborhoods over time in our longitudinal analysis. To do so we essentially model mortality rates as dependent on the underlying social and economic vulnerabilities of interest at one level of aggregation. This approach explores only one very distinct aspect of the complex behavior of human systems. For example, it does not consider how 
population health itself may in turn affect the underlying socioeconomic vulnerabilities (e.g., healthier populations may be better able to improve overall economic status), that different vulnerabilities may interact (e.g., populations characterized both by lower aggregate incomes and lower aggregate educational attainments may be worse off than populations characterized by one or the other of these vulnerabilities), or that the relationship between underlying population vulnerabilities and intermittent stressors may change over time. In effect, in this paper we are presenting an overall heuristic that may help explain patterns of population health but are assessing one limited aspect of these patterns (variability) in a specific circumstance, i.e., in urban neighborhoods over the past decade. Further work that aims to explore elements of the heuristic framework presented here will need to rely more heavily on methods that allow the modeling of complex systems.

It has been suggested that "the most difficult general problem of contemporary science is how to deal with complex systems as wholes" (Levins, 1974). However, specific methods have been proposed that suggest how we may deal with complex systems as a whole and may present promising avenues for further analyses (Levins, 1974). We note that the model presented here is not meant to represent direct causal relations between the constructs identified, but rather is meant to show how these constructs together are relevant determinants of population health. More detailed causal diagrams incorporating the inter-relations of the various domains discussed here would be necessary in order to guide public health prediction. Multilevel linear models (DiezRoux, 2000) may also allow the exploration of the dynamics of populations that are nested within other population groups and improve our understanding of the relationship among different population groups.

These observations have a number of implications for public health practice and research. The methods used here, if replicated in other work and extended to assess some of the considerations discussed above, can be used to conduct more insightful community health assessments and in turn, more appropriately guide public health planning. Examining variability in rates in addition to their absolute levels can reflect underlying socioeconomic vulnerability that extends public health surveillance beyond the capabilities of extant methods. Typical community health assessment considers the average rates of specific morbidities and mortalities in populations to identify areas in need of services or programs. In contrast, a "vulnerability" model of health assessment suggests that certain neighborhoods are vulnerable to poor health even if, at one point in time, health status in that neighborhood is not worse than in other neighborhoods. In fact, at a single point in time, health in some vulnerable neighborhoods may be as good as, or better than, health in less vulnerable neighborhoods. However, in response to new stressors these populations will likely experience elevated rates of disease. The policy implication is that such neighborhoods require public health attention regardless of the point prevalence of key health indicators.

These observations suggest that populations that are similarly characterized by underlying vulnerabilities behave very differently. There might be tremendous insight into the health of populations gained by studying why populations that share underlying vulnerabilities, such as poverty, often have quite different health outcomes. Our model suggests that the reasons for these differences include the differential distribution of underlying capacities or of unequal exposures to intermittent stressors and protective events. Some populations with high levels of vulnerability along one axis had lower mortality rates than many other neighborhoods, including those with lower levels of vulnerability (see, for example, Fig. 1). Such populations may provide insight into capacities that counteract vulnerabilities. Currently, our understanding of the scope of socioeconomic vulnerabilities remains limited and our understanding of underlying capacities even more so. Factors such as social capital are likely candidates for capacities that positively affect population health, but more work is required to fully identify the capacities that may determine health and that may be amenable to public health intervention.

Recently, national public health discourse has focused extensively on the problem of health disparities in the United States and on efforts to reduce these disparities (Department of Health and Human Services, 2000; Whaley, 2003; Andrews, Graham-Garcia, \& Raines, 2001; Elster, Jarosik, VanGeest, \& Fleming, 2003). This discussion of disparities focuses on making health parameters in specific populations (defined by race/ ethnicity or socioeconomic status) comparable. As such, there have been several federal initiatives (and numerous research efforts) that provide interventions aimed at improving the health status of these specific populations. For example, public health interventions aimed at improving morbidity among African-American women have included promotion of healthier diets and increased exercise (Story et al., 2003; Rimmer, Silverman, Braunschweig, Quinn, \&Liu, 2002). However, our model suggests that insofar as specific groups characterized by race/ethnicity are human populations, in the absence of a change in the underlying vulnerabilities, interventions that are essentially "protective events" added to the proposed model will not likely have a substantial impact on the underlying vulnerabilities and capacities of the population. We would not expect such interventions to improve population health in the long term. This may explain why the positive effects of many public health interventions are unsustainable beyond the duration of the intervention itself (Shediac-Rizkallah \& Bone, 1998). 
This work suggests that in the absence of efforts to alter vulnerabilities and capacities some human populations will continue to have worse than average health despite the best public health interventions.

In conclusion, we suggest that the study of the health of human populations is incomplete without an appreciation of the ecology of human health. We propose a simple model for considering the underlying vulnerabilities and capacities and intermittent stressors and protective events that shape population health. Further work is needed to identify features of populations that represent these particular elements and to identify methods that can usefully model population dynamics for the eventual purpose of public health prediction.

\section{Acknowledgements}

The authors are grateful to Dr. Richard Levins and Dr. Tamara Awerbuch for early discussions that led to the work described here and for comments on an earlier version of this manuscript. We are indebted to three reviewers who provided detailed comments on an earlier version of this manuscript and in particular to reviewer B who helped us clarify several of the concepts presented here. Some of this work was presented at the Harvard University School of Public Health Population and International Health Mathematical Modeling seminar series in Boston, MA, November 2003 and at the Society for Epidemiologic Research Annual Meeting in Salt Lake City, UT, June 2004.

\section{References}

Adler, N. E., Boyce, T., Chesney, M. A., Cohen, S., Folkman, S., Kahn, R. L., \& Syme, S. L. (1994). Socioeconomic status and health. The challenge of the gradient. American Psychologist, 49, 15-24.

Andrews, J. O., Graham-Garcia, J., \& Raines, T. L. (2001). Heart disease mortality in women: racial, ethnic, and geographic disparities. The Journal of Cardiovasc Nursing, $15,83-87$.

Bankoff, G. (2003). Constructing vulnerability: the historical, natural and social generation of flooding in metropolitan Manila. Disasters, 27, 224-238.

Breusch, T. S., \& Pagan, A. R. (1979). A simple test for heteroskedasticity and random coefficient variation. Econometrica, 47, 1287-1294.

Cohen, S., \& Hamrick, N. (2003). Stable individual differences in physiological response to stressors: implications for stress-elicited changes in immune related health. Brain, Behavior, and Immunity, 17, 407-414.

Curtis, S., \& Rees Jones, I. (1998). Is there a place for geography in the analysis of health inequality? In M. Bartley, D. Blane, \& G. Davey Smith (Eds.), The sociology of health inequalities. Oxford: Blackwell Publishers.
Department of Health and Human Services. (2000). Eliminating racial and ethnic disparities in health. http://raceandhealth.hhs.gov/sidebars/sbinitOver.htm (3 December 2003).

Diez-Roux, A. V. (2000). Multilevel analysis in public health research. Annual Review of Public Health, 21, 171-192.

Elster, A., Jarosik, J., VanGeest, J., \& Fleming, M. (2003). Racial and ethnic disparities in health care for adolescents: a systematic review of the literature. Archives of Pediatric and Adolescent Medicine, 157, 867-874.

Galea, S., Ahern, J., Vlahov, D., Coffin, P. O., Fuller, C., Leon, A. C., \& Tardiff, K. (2003a). Income distribution and risk of fatal drug overdose in New York City neighborhoods. Drug and Alcohol Dependence, 70, 139-148.

Galea, S., Vlahov, D., Resnick, H., Ahern, J., Susser, E., Gold, J., Bucuvalas, M., \& Kilpatrick, D. (2003b). Trends in probable posttraumatic stress disorder in New York City after the September 11 terrorist attacks. American Journal of Epidemiology, 158, 514-524.

Geronimus, A. T., Bound, J., \& Waidmann, T. A. (1999). Poverty, time, and place: variation in excess mortality across selected US populations, 1980-1990. Journal of Epidemiological Community Health, 53, 325-334.

Healy, J. D. (2003). Excess winter mortality in Europe: a cross country analysis identifying key risk factors. Journal of Epidemiological and Community Health, 57, 784-789.

Heath, A. C., \& Nelson, E. C. (2002). Effects of the interaction between genotype and environment. Research into the genetic epidemiology of alcohol dependence. Alcohol Research \& Health: The Journal of the National Institute on Alcohol Abuse and Alcoholism, 26, 193-201.

Hillemeier, M. M., Lynch, J., Harper, S., Raghunathan, T., \& Kaplan, G. A. (2003). Relative or absolute standards for child poverty: a state-level analysis of infant and child mortality. American Journal of Public Health, 93, 652-657.

Kaplan, G. A., Pamuk, E. R., Lynch, J. W., Cohen, R. D., \& Balfour, J. L. (1996). Inequality in income and mortality in the United States: analysis of mortality and potential pathways. British Medical Journal, 312, 999-1003.

Karpati, A., Galea, S., Awerbuch, T., \& Levins, R. (2002). Variability and vulnerability at the ecologic level: implications for understanding the social determinants of health. American Journal of Public Health, 92, 1768-1772.

Kawachi, I., Kennedy, B. P., Lochner, K., \& Prothrow-Stith, D. (1997). Social capital, income inequality, and mortality. American Journal of Public Health, 87, 1491-1498.

Koopman, J. S., \& Longini, I. M. (1994). The ecological effects of individual exposures and nonlinear disease dynamics in populations. American Journal of Public Health, 84, 836-842.

Koopman, J. S., \& Lynch, J. W. (1999). Individual causal models and population system models in epidemiology. American Journal of Public Health, 89, 1170-1174.

Latkin, C. A., Forman, V., Knowlton, A., \& Sherman, S. (2003). Norms, social networks, and HIV-related risk behaviors among urban disadvantaged drug users. Social Science \& Medicine, 56, 465-476.

Levins, R. (1974). The qualitative analysis of partially specified systems. Annals of the New York Academy of Sciences, 231, 123-138.

Levins, R. (1975). Evolution in communities near equilibrium. In M. L. Cody, \& J. M. Diamond (Eds.), Ecology and 
evolution of communities (pp. 16-50). Cambridge, UK: The Belknap Press of Harvard University Press.

Levins, R., \& Lopez, C. (1999). Toward an ecosocial view of health. International Journal of Health Services, 29, 261-293.

Link, B. G., \& Phelan, J. C. (2000). Evaluating the fundamental cause explanation for social disparities in health. In C. E. Bird, P. Conrad, \& A. M. Fremont (Eds.), Handbook of medical sociology: 5th ed (pp. 33-46). New Jersey: Upper Saddle River.

Marmot, M. G., Kogevinas, M., \& Elston, M. A. (1987). Social/economic status and disease. Annual Review of Public Health, 8, 111-135.

Marzuk, P. M., Tardiff, K., Leon, A. C., Hirsch, C. S., Stajic, M., Portera, L., \& Hartwell, N. (1997). Poverty and fatal accidental drug overdoses in New York City. American Journal of Drug Alcohol Abuse, 23, 221-228.

Maynard, R., Krewski, D., Burnett, R. T., Samet, J., Brook, J. R., Granville, G., \& Craig, L. (2003). Health and air quality: directions for policy-relevant research. Journal of Toxicology and Environmental Health, Part A, 66, 1891-1904.

McKeehan, I. V. (2000). A multilevel city health profile of Moscow. Social Science \& Medicine, 51, 1295-1312.

Nelson, D. (1990). Mitigating disasters: power to the community. International Nursing Review, 37, 371.

O'Campo, P. (2003). Invited commentary: advancing theory and methods for multilevel models of residential neighborhoods and health. American Journal of Epidemiology, 157, 9-13.

Philippe, P., \& Mansi, O. (1998). Nonlinearity in the epidemiology of complex health and disease processes. Theoretical Medicine and Bioethics, 19, 560-591.

Rimmer, J. H., Silverman, K., Braunschweig, C., Quinn, L., \& Liu, Y. (2002). Feasibility of a health promotion intervention for a group of predominantly African American women with type 2 diabetes. The Diabetes Educator, 28, 571-580.

Robert, S. A. (1999). Socioeconomic position and health: the independent contribution of community socioeconomic context. Annual Review of Sociology, 25, 489-516.
Sampson, R. J., Morenoff, D., \& Gannon-Rowley, T. (2002). Neighborhood effects; social processes and new directions in research. Annual Review of Sociology, 28, 443-478.

Sampson, R. J., Raudenbush, S. W., \& Earls, F. (1997). Neighborhoods and violent crime: a multilevel study of collective efficacy. Science, 277, 918-924.

Schmalhausen, I. I. (1949). Factors of evolution. Philadelphia, PA: Blakiston Company.

Shediac-Rizkallah, M. C., \& Bone, L. R. (1998). Planning for the sustainability of community-based health programs: conceptual frameworks and future directions for research, practice, and policy. Health Education Research, 13, 87-108.

Shi, L. (2000). Vulnerable populations and health insurance. Medical Care Research and Review, 57, 110-134.

Stergiopoulos, V., \& Herrmann, N. (2003). Old and homeless: a review and survey of older adults who use shelters in an urban setting. Canadian Journal of Psychiatry, 48, 374-380.

Story, M., Sherwood, NE., Himes, J. H., Davis, M., Jacobs, D. R., Jr., Cartwright, Y., Smyth, M., \& Rochon, J. (2003). An after-school obesity prevention program for African-American girls: the Minnesota GEMS pilot study. Ethnicity and Disease, 13(Suppl.), 54-64.

Subramanian, S. V., \& Kawachi, I. (2004). Income inequality and health: what have we learned so far? Epidemiologic Reviews, 26, 78-91.

Subramanian, S. V., Kim, D. J., \& Kawachi, I. (2002). Social trust and self-rated health in US communities: a multilevel analysis. Journal of Urban Health, 79(Suppl.), 21-34.

Tardiff, K. J., Gross, E. M., \& Messner, S. (1986). A study of homicides in Manhattan, 1981. American Journal of Public Health, 76, 139-143.

Turner, B. L., II, Kasperson, R. E., Matson, P. A., McCarthy, J. J., Corell, R. W., Christensen, L., Eckley, N., Kasperson, J. X., \& Luers, A. (2003). A framework for vulnerability analysis in sustainability science. Proceedings of the National Academy of Sciences of the United States of America, 100, 8074-8079.

Whaley, A. L. (2003). Ethnicity/race, ethics, and epidemiology. Journal of the National Medical Association, 95, 736-742. 